\title{
Formation of Bimetallic Nanoparticle Arrays and Evidence for their Stability at High Temperature under Gas Pressure in the Environmental TEM
}

\author{
Eric Ehret ${ }^{1}$, Francisco J. Cadete Santos Aires $^{1,2}{ }^{,}$Bruno Domenichini $^{3}$, Laurence Burel ${ }^{1}$ and Thierry \\ Epicier $^{1}$ \\ ${ }^{1}$ IRCELYON, UMR 5256 CNRS, Univ. Claude Bernard Lyon 1, 69626 Villeurbanne cedex, France. \\ ${ }^{2}$ LCR, National Research Tomsk State University, Tomsk 634050, Russian Federation. \\ ${ }^{3}$ ICB, UMR 6303 CNRS, Univ. Bourgogne Franche-Comté, 21078 Dijon cedex, France.
}

Supported catalysts are generally composed of, at least, one nanometric active phase deposited and/or synthesized at the surface of a convenient nanometer/micrometer powder support. As such these are rather complex systems with many variable parameters: dispersion on the support, size distribution, morphology, structure, defects, variable chemical phases, variable exposed facets of the active phases, interaction with the support, etc. It is thus quite cumbersome to correlate a catalytic behavior with specific physicochemical properties. The synthesis of nanometric particles (NPs) with well controlled characteristics is thus crucial to better understand the behavior of the active phase during reaction and to better correlate its behavior with its catalytic performance. The di-block copolymer approach is well suited to obtain well controlled (size, ordered arrays) supported NPs which can be used as supported metallic catalysts on both flat (model catalysts) and powder (realistic catalysts) supports [1]. In this approach, an amphiphilic di-block copolymer dissolved in toluene yields a system of inverse micelles. We can then metallize the core of these micelles by introducing metal salts. Such systems can then be deposited on flat surfaces by dip or spin coating for instance. We have already extended this method to the synthesis of bimetallic model catalysts [2]. However, many questions are still open concerning, for instance, the formation of unique NPs at the core from the pre-formed bimetallic seeds (Fig. 1b-d) or the stability of the arrays of bimetallic NPs (Fig. 1a) with increasing temperature in presence of reactive gases.

In order to gain insight on such issues we have used an Environmental TEM (Titan ETEM G2 from FEI/ThermoFisher Scientific) operated at $300 \mathrm{kV}$ under gas pressures up to 20 mbar. To vary the temperature, we have used a WildFire support holder with dedicated heating microchips (DENS Solutions) capable of reaching temperatures up to $1300^{\circ} \mathrm{C}$. On top of the latter an Au-rich AuPd coremetallized PS- $b$-P2VP micellar solution was deposited by spin-coating. Special care was taken to minimize/prevent any influence of the electron beam on the observed events; for instance, a new area was systematically used before each increasing step in temperature (Fig. 2). Temperature treatment under oxygen is crucial both to achieve the formation of unique NPs on the micellar core and to eliminate the copolymer. We could see that the seeds within the micellar cores begin to sinter at around $350^{\circ} \mathrm{C}$ (consistent with the temperature at which the polymers are decomposed and gasified) and the unique NPs are obtained at around $500^{\circ} \mathrm{C}$ (Fig. 2). The network is remarkably stable above $500^{\circ} \mathrm{C}$ and up to $800 / 900^{\circ} \mathrm{C}$ (Figure 3); around $1000^{\circ} \mathrm{C}$, when the temperature approaches the melting temperature of $\mathrm{Au}\left(1064^{\circ} \mathrm{C}\right)$, the NPs begin to decompose (Fig. 3). These observations clearly open the possibility of using these structures under rather extreme catalytic conditions (and thus widens the range of catalytic applications); the stability of the arrays appears to be (only) dependent on the intrinsic properties of the metals used [3].

\section{References:}

[1] B Roldan Cuenya, Accounts of Chemical Research 46 (2013) 1682. 
[2] E Ehret et al, Nanoscale 7 (2015) 13239.

[3] The authors acknowledge the French Microscopy and Atom probe network (METSA) and the Consortium Lyon - St-Etienne de Microscopie (CLYM) for supporting this work.
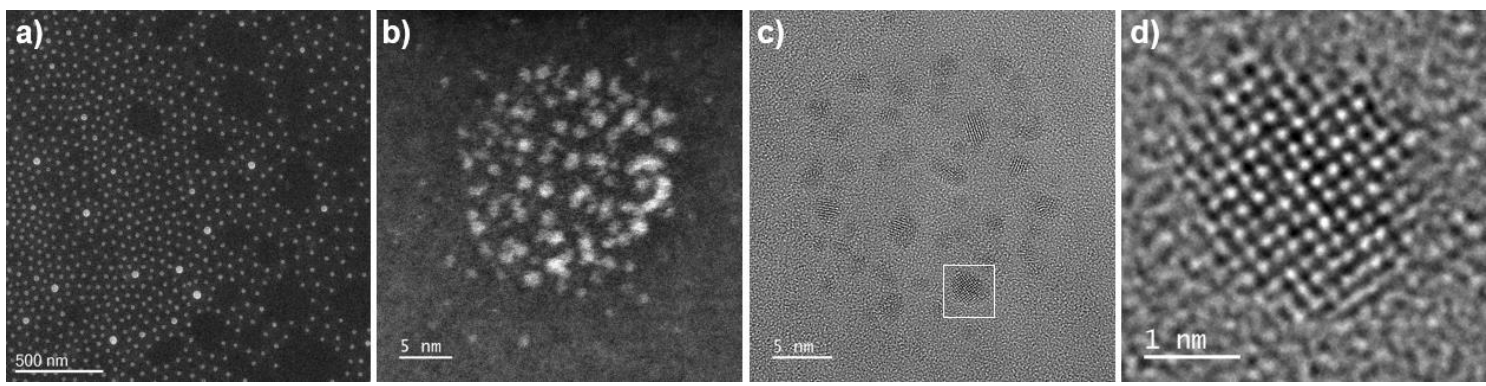

Figure 1. Array obtained by spin-coating of a micellar solution of di-block copolymer PS- $b$-P2VP containing $\mathrm{Pd}$ and $\mathrm{Au}$ seeds within the P2VP cores. (a) general view (ADF), (b,c) detailed views of micellar cores containing bimetallic seeds $(b-A D F, c-B F)$, (d) shows a HR BF image of a bimetallic seed (frame in c) and oriented along the [001] fcc zone axis.



area $22: 50^{\circ} \mathrm{C}, 1.5 \mathrm{mbar}_{2}$

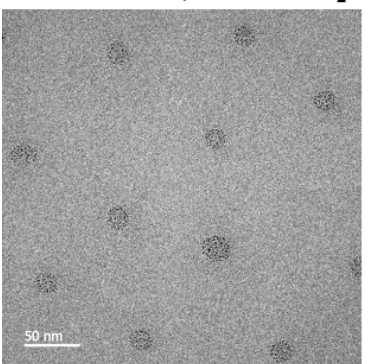



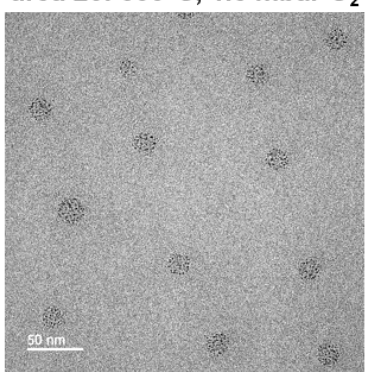

area $27: 350^{\circ} \mathrm{C}, 1.8 \mathrm{mbar}_{2}$
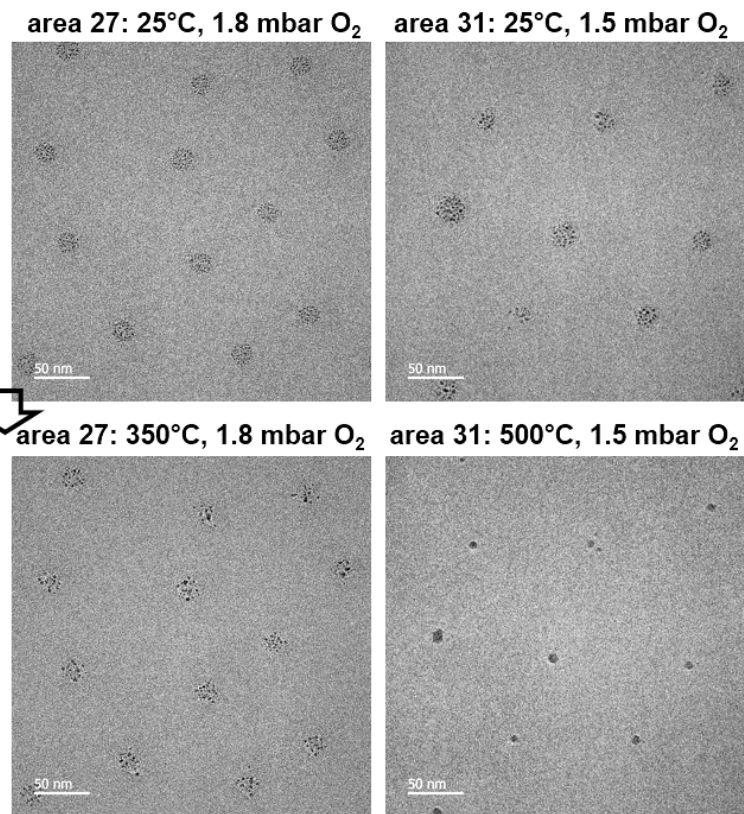

area $31: 500^{\circ} \mathrm{C}, 1.5 \mathrm{mbar}_{2}$

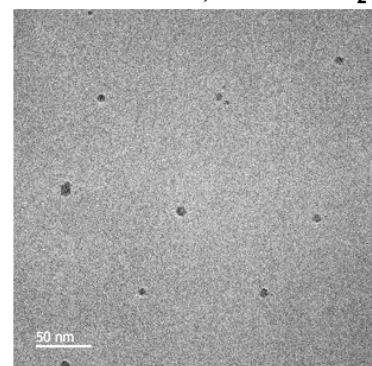

Figure 2. Bimetallic seeds within the micellar core begin to sinter around $350^{\circ} \mathrm{C}$ and fully form NPs around $500^{\circ} \mathrm{C}$. Each temperature step is made on a fresh region to avoid eventual electron beam damage.
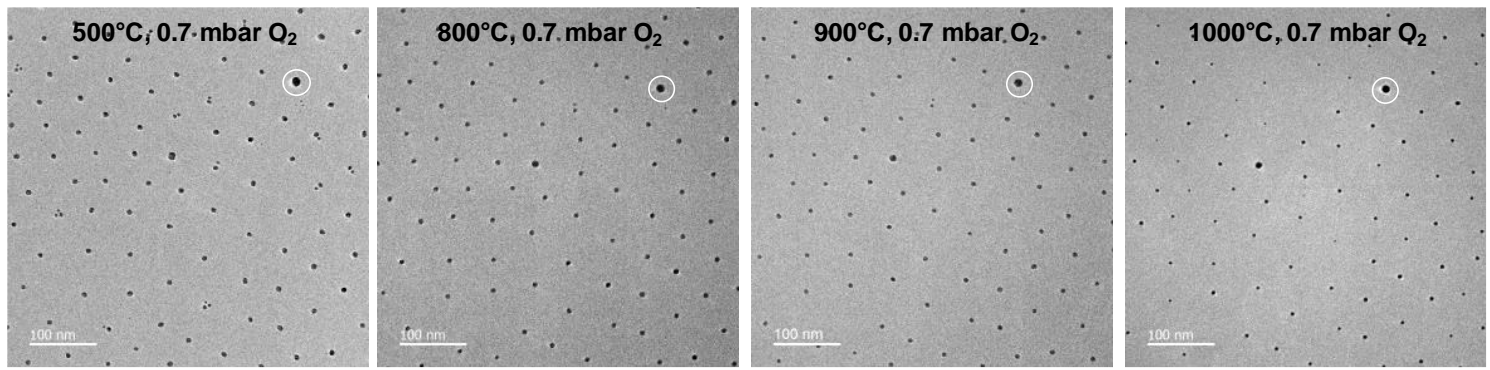

Figure 3. Stability of the network up to $800 / 900^{\circ} \mathrm{C}$ under oxygen. Approaching the melting point of $\mathrm{Au}$ $\left(1064^{\circ} \mathrm{C}\right)$ the NPs begin to decompose. 